\title{
Laboratory testing of tennis strings
}

\author{
Rod Cross, Crawford Lindsey ${ }^{1}$ and Daniel Andruczyk \\ Physics Department, University of Sydney, Sydney, NSW, Australia \\ ${ }^{1}$ USRSA, Del Mar, California, USA
}

\begin{abstract}
Most tennis strings have a performance rating of almost 10 out of 10 if one can believe the manufacturers' claims. Laboratory tests of tennis strings provide a different picture. The test methods and the results of testing 90 different strings are described. This type of information is needed if players, coaches and stringers wish to make an informed comparison between different strings, and it is also needed if one wishes to model the interaction between a tennis racket and a tennis ball.
\end{abstract}

Keyzords: tennis, strings, tension, stiffness, elongation, friction break

\section{Introduction}

Tennis balls are subject to a number of rules and test procedures before they are approved for tournament use, but there are no standard tests or rules governing the properties of tennis strings. Strings can be as thick or thin, as rough or smooth and as stiff or elastic as the manufacturer can make them. As a result, there are many hundreds of varieties on the market, and there are no approved methods available to compare one against the other. The only guide to the properties of strings, apart from playtest reports in popular magazines and some early test results by Calder et al. (1987), are the ratings on the packet provided by the manufacturers. It is not surprising that nearly all strings have playability or performance ratings of nearly 10 out of 10 . From a scientific and engineeering point of view, these ratings are essentially worthless since the test methods are not stated and the results cannot be expressed in SI units. In this

Correspondence address:

Rod Cross, Physics Department, University of Sydney, Sydney, NSW 2006, Australia.

Fax: +61293517727.

E-mail: cross@physics.usyd.edu.au paper, a number of test methods and sample results are described, and a summary is given of the results of testing 90 different strings. The string properties measured included (a) elongation as a function of string tension, (b) dynamic stiffness, (c) loss in tension as a function of time and as a result of repeated impacts, (d) energy loss during an impact and (e) the coefficient of friction between the string and the cloth of a tennis ball. All strings tested were of the same nominal diameter (16 gauge, nominally $1.29 \mathrm{~mm}$ ) but the actual diameters varied from $1.25 \mathrm{~mm}$ to $1.40 \mathrm{~mm}$. The variation in diameter along any given string was typically about $\pm 0.01 \mathrm{~mm}$. An important property that was not measured in this survey was the durability of the string.

Under a constant static load, the elongation of a tennis string increases with time (Brody 1987). This effect is known as creep. The rate of creep decreases with time, but the elongation continues to increase for as long as one is willing to take measurements. Such materials are said to be viscoelastic, meaning that they are both viscous and elastic. A stress vs. strain curve therefore depends on the rate of application of the stress or the strain. For example, a string might elongate by $10 \%$ if it is stretched to a tension of $300 \mathrm{~N}$ at a rate of $100 \mathrm{~mm} \mathrm{~min}^{-1}$, or by $8 \%$ at $200 \mathrm{~mm} \mathrm{~min}^{-1}$. These 
strain rates are typical of those used to test engineering materials in devices such as an Instron pull test machine. Under actual playing conditions, where the ball impacts the strings for a period of only about $5 \mathrm{~ms}$, the rate of stretch is typically about $40000 \mathrm{~mm} \mathrm{~min}^{-1}$. For such a short duration impact, the amount of creep is relatively small and the elongation does not depend significantly on the impact duration. However, a small amount of creep can still occur even during a very short impact, especially in a string that has not previously been stretched. As a result, the strings in a racket will lose tension after each impact, especially during the first few impacts on a freshly strung racket. In this paper, the elongation was measured under dynamic conditions, for an impact of about $30 \mathrm{~ms}$ duration. All strings were tested under identical conditions, starting with a fresh, previously unstretched length of string. The strings were tested in an air-conditioned laboratory, at a temperature of $21^{\circ} \mathrm{C}$.

An effect that is complementary to creep occurs in a strung racket. If a string is stretched to a given length and then clamped at this length, the tension immediately starts to decrease, due to the gradual breaking of bonds between long-chain molecules. This effect is known as stress relaxation. The rate at which the string loses tension decreases with time, but the tension continues to decrease for as long as one is willing to take measurements. The magnitude of the effect is larger than one might expect and it is considerably larger than the estimates we obtained from several experienced racket stringers. For example, if a racket is strung at a tension of $28 \mathrm{~kg}$, the tension will drop typically to about $24 \mathrm{~kg}$ within $20 \mathrm{~min}$, which is about the time it takes to string a racket. After $24 \mathrm{~h}$, the tension is typically about $20 \mathrm{~kg}$. After using the racket for one or two sets, the tension will drop a further one or two $\mathrm{kg}$ in most strings, due to repeated stretching of the string to tensions above $28 \mathrm{~kg}$. The string tension may also be affected by deformation of the frame during the stringing process (Casolo \& Lorenzi 2000) and the tension may not be the same in every string due to friction between each string and the grommet holes.

\section{Experimental procedures}

Measurements of elongation, tension loss and impact tests were undertaken using the apparatus shown in Fig. 1. This apparatus was designed to monitor changes in string tension with time and with repeated impacts, while leaving the clamped length of the string fixed. The string was clamped in metal jaws separated by a distance of $320 \mathrm{~mm}$ prior to stretching, and then stretched to a tension of $20 \mathrm{~kg}(196 \mathrm{~N})$ for a few seconds in order to measure the elongation. The tension was adjusted manually, by rotating the tensioning nut with a spanner. The elongation was determined by marking the string with a felt pen and measuring the extension with a ruler. As described above, this elongation measurement is not very accurate since it depends on the rate at which the string is stretched. However, it provides an interesting comparison between different strings. The tension was measured using a commercial load cell and electronic indicator calibrated to read up to $100 \mathrm{~kg}$ with a resolution of $0.01 \mathrm{~kg}$ and with automatic
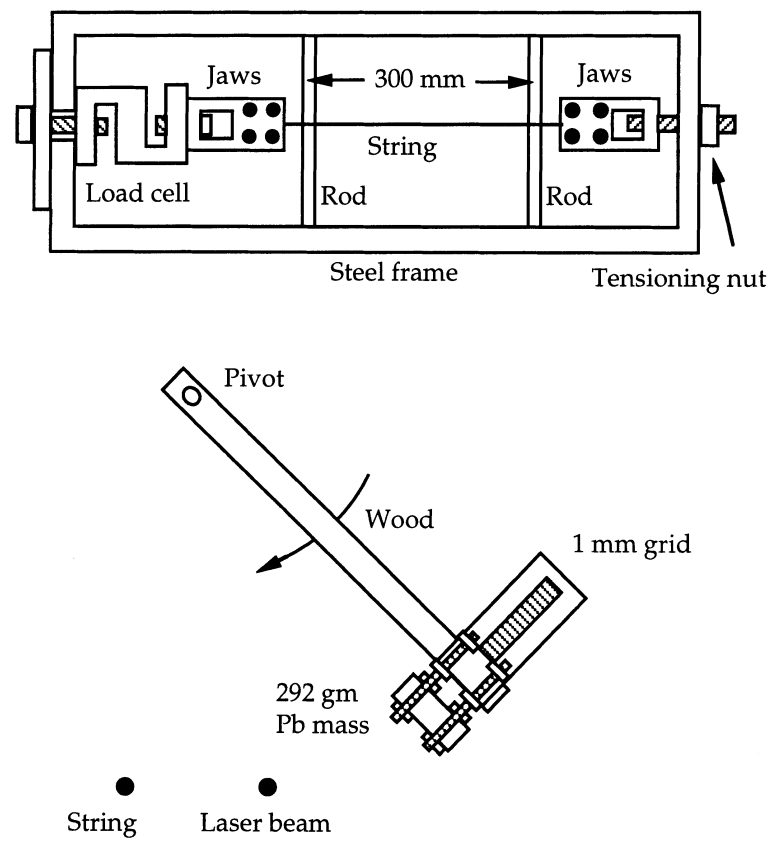

Figure 1 Apparatus used to measure string tension and the transverse displacement of a string when subjected to a hammer impact. 
zero adjustment. As well as a digital readout, the indicator had a $0-10 \mathrm{~V}$ analogue output which was connected to a storage oscilloscope and a data acquisition system.

After recording the elongation at $20 \mathrm{~kg}$, the tension was increased to $28 \mathrm{~kg}$ and held at this tension for $10 \mathrm{~s}$ by manual adjustment of the tensioning nut. This simulates the procedure commonly used when stringing a racket, where the string is tensioned for about $10 \mathrm{~s}$ before the stringer applies a clamp to hold the string in place. For strings that lost tension slowly, this required a slow increase in elongation in order to maintain constant tension. Some strings lost tension rapidly, in which case it was necessary to stretch the string at a much faster rate to maintain the tension at $28 \mathrm{~kg}$. After this 10 -s period, no further adjustment of the tensioning nut was made, and the tension was allowed to decrease for a period of $1000 \mathrm{~s}$ (16.7 min). During this time, the elongation at $28 \mathrm{~kg}$ was measured, to compare with the elongation at $20 \mathrm{~kg}$, and the tension was recorded at $1 \mathrm{~s}$ intervals with the data acquisition system. In previous tests on a number of other strings, it was established that a plot of tension vs. $\log$ (time) is linear after the first $100 \mathrm{~s}$, even over a period of several days. The test period was therefore shortened to $1000 \mathrm{~s}$ for the 90 strings in the present study.

A number of tests were made on the jaws used to hold the string at each end. In an attempt to minimise damage in the metal jaws, each end of the string was protected by a layer of plastic heatshrink. However, it was found that the heatshrink had its own rate of creep, so this technique was abandoned. Commercial stringer's clamps were also tried, but these did not clamp the string firmly enough. Stringer's clamps leave no marks on the string, and are adequate for normal use, but the string pulled through the clamps by about $1 \mathrm{~mm}$ at the entry point (but not the exit point) when tensioned to $28 \mathrm{~kg}$ and by an additional $1 \mathrm{~mm}$ during impact tests. The clamps used in the present study were constructed from a fine file, cut into $38 \mathrm{~mm}$ lengths and clamped firmly onto the string with a screw in each corner. After removing the string from the clamps, the string was left slightly but permanently squashed and indented with teeth marks from the clamps. However, the damage to the string had no effect on the results. Provided the clamps were tightened sufficiently, it was found that the elongation and tension loss results were reproducible and independent of the clamping force. Furthermore, the results were the same as those measured in a fully strung racket. In the latter case, the string tension in two cross strings was measured by passing the string through an external adaptor attached to both the racket frame and the load cell.

After allowing the string to relax for $1000 \mathrm{~s}$, it was then subjected to 10 impacts at the centre of the string using a hammer incident at $2.63 \mathrm{~m} \mathrm{~s}^{-1}$ and at right angles to the string. Two cylindrical rods were located near each of the jaws, as shown in Fig. 1, to restrict the transverse motion of the string to a $300-\mathrm{mm}$ region between the rods. This ensured that tension was applied to the load cell along its axis, and it minimised possible damage to the string at the entry point to the jaws. The hammer consisted of a lead block of mass $292 \mathrm{~g}$ mounted at the bottom end of a light wooden beam. The beam was pivoted at the top end by means of a ball race to minimise friction at the pivot point. The hammer was allowed to swing into the string, through a fixed angle, as a pendulum. Under normal conditions, the strings of a racket experience a peak transverse force of up to about $1500 \mathrm{~N}$. Such a force, acting on a ball of mass $57 \mathrm{~g}$ over a period of about $5 \mathrm{~ms}$, is required to change its velocity from $+30 \mathrm{~m} \mathrm{~s}^{-1}$ to $-30 \mathrm{~m} \mathrm{~s}^{-1}$. The force is distributed over all the strings, but if one assumes that the brunt of the force is shared mainly by five mains and five cross strings, then the peak force on each string is about $150 \mathrm{~N}$. In the apparatus shown in Fig. 1, the total effective mass of the $292 \mathrm{~kg}$ lead block, the wood beam and the attached optical grid was $0.45 \mathrm{~kg}$. The hammer changed its velocity from $+2.63 \mathrm{~m} \mathrm{~s}^{-1}$ to about $-2.5 \mathrm{~m} \mathrm{~s}^{-1}$ over a period of about $30 \mathrm{~ms}$, giving a peak force between 120 and $200 \mathrm{~N}$ on the string, depending on its stiffness.

Since a single string has a much lower transverse stiffness than the strings of a fully strung racket, it 
is difficult to simulate, with a single string, the impact conditions encountered during normal use. To maintain the same peak force and impact duration, it would be necessary to impact the string with a projectile of mass much smaller than that of a tennis ball, travelling at a speed much higher than the normal speed of a tennis ball. Alternatively, one can maintain the same peak force using a low speed hammer of mass larger than that of a tennis ball. In this case, the impact duration is longer than normal, but this has the advantage of simulating the cumulative effect of a number of impacts each of duration $5 \mathrm{~ms}$.

Measurements of the velocity of the hammer and the transverse displacement of the string were made by passing a laser beam through an optical grid attached to the hammer, as shown in Fig. 1. The grid consisted of $501 \mathrm{~mm}$ thick parallel lines separated by $1 \mathrm{~mm}$, photocopied onto an overhead transparency and mounted in a light aluminium frame. Because the beam diameter was slightly larger than $1 \mathrm{~mm}$, the detected laser signal consisted of a series of sinusoidal fringes as described below.

\section{Typical results}

Figure 2 shows string tension as a function of time for a natural gut and a polyester string initially

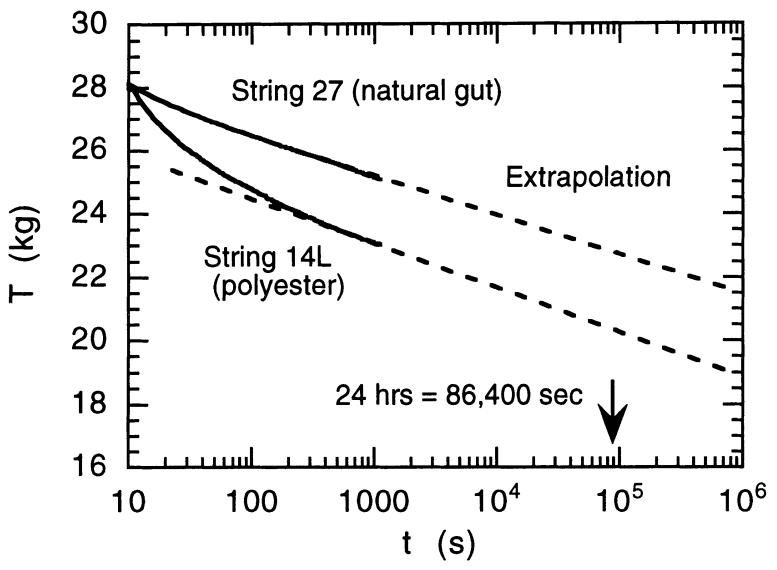

Figure 2 Tension vs. $\log$ (time) for two different strings initially tensioned to $28 \mathrm{~kg}$ for $10 \mathrm{~s}$ prior to clamping. tensioned to $28 \mathrm{~kg}$. Natural gut has one of the lowest rates of tension loss (some kevlar strings are lower) while all polyester strings lose tension rapidly. The rate at which a string loses tension can be decreased by holding the initial tension above $20 \mathrm{~kg}$ for a period of about $30 \mathrm{~s}$, or by prestretching the string one or more times (Cross 2000a). This is generally not a practical proposition considering that a racket stringer would have to charge an additional hourly rate to string the racket. Alternatively, the string can be tensioned at a higher initial tension to compensate for tension loss after clamping. The effect of stretching a string to $28 \mathrm{~kg}$ for various time intervals, before clamping the string, is shown in Fig. 3. The effect is quite pronounced, and it also affects tension loss during subsequent impacts. Because the tension was adjusted manually for $10 \pm 1 \mathrm{~s}$ prior to clamping, rather than being controlled electronically, it was difficult to maintain the tension accurately at $28 \mathrm{~kg}$ for precisely $10 \mathrm{~s}$ during the prestretch. The tension loss measurements in this paper are therefore reliable to only about $\pm 0.1 \mathrm{~kg}$, but this was adequate for the purpose of comparing different strings.

The effect of a series of 10 hammer impacts on string tension is shown in Fig. 4. The tension rises during each impact by an amount that depends on the elasticity of the string. The rise in tension during each impact is not properly recorded in

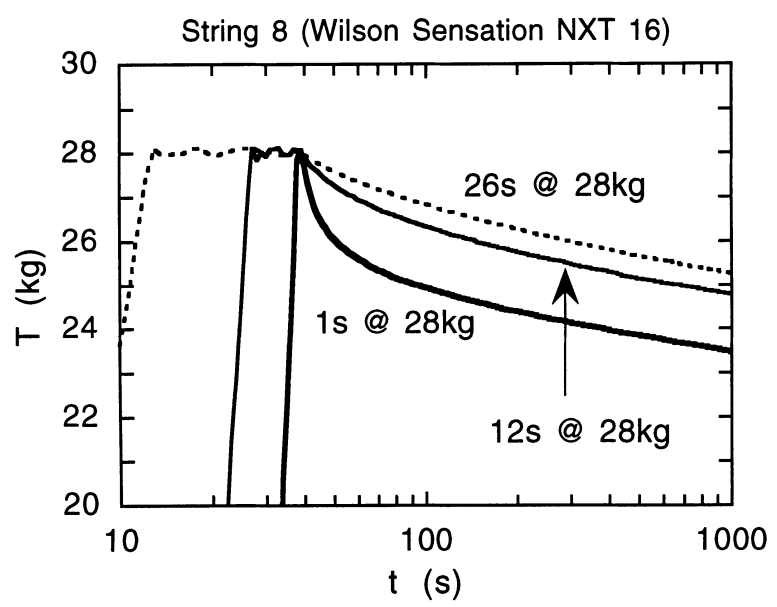

Figure 3 Tension vs. $\log$ (time) for a string tensioned to $28 \mathrm{~kg}$ for several different time intervals prior to clamping. 


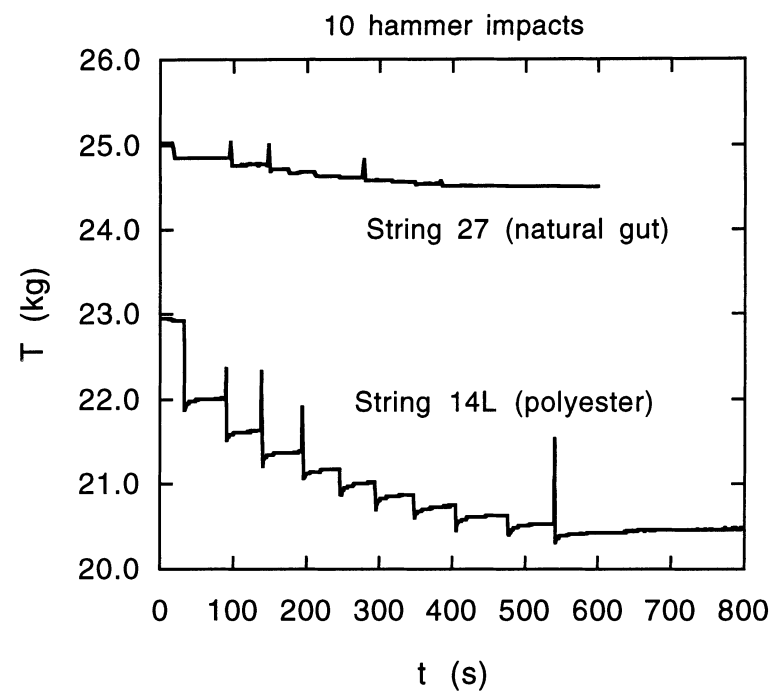

Figure 4 Effect of a series of 10 hammer impacts on string tension for the strings shown in Fig. 2. Figure 4 is a continuation of Fig. 2, the first impact commencing at $t=1020 \mathrm{~s}$ (re-defined as $t=0$ in Fig. 4).

Fig. 4 because the data was captured at only one point every second. The tension rises by about $9 \mathrm{~kg}$ for natural gut, about $18 \mathrm{~kg}$ for nylon strings, about $22 \mathrm{~kg}$ for polyester strings and about $45 \mathrm{~kg}$ for kevlar strings. The effect is the same as that for a static increase in tension, in that the tension starts to decrease rapidly as soon as the tension is increased. As a result, the tension immediately after an impact is less than that before the impact, particularly after the first impact. Successive impacts result in successively smaller drops in tension. The decrease in tension after each impact therefore depends on the previous history of the string, and it also depends on the magnitude of the impact. If the experiment is repeated at a lower hammer speed or with a lighter hammer, then the net loss in tension after a series of 10 impacts is reduced. Conversely, the loss in tension is greater with a heavier hammer.

An interesting effect occurs between impacts, in that there is a slow recovery of tension in most strings. This effect can be seen in Fig. 4 for the polyester string. Given that a decrease in tension can be explained by the breaking of molecular bonds, then an increase in tension is presumably due to the formation of new bonds. Alternatively, one can describe the string as having a memory. If the tension is held at, say, $28 \mathrm{~kg}$ for a few minutes, and is then decreased quickly to, say, $15 \mathrm{~kg}$ and clamped at this value, then the tension will immediately start to rise back towards $28 \mathrm{~kg}$. The tension will settle at a steady value, about $18 \mathrm{~kg}$, when the rate of formation of new bonds is balanced by the rate at which old bonds break.

The rise in tension during an impact, and the corresponding laser signal used to monitor the displacement of the string, is shown in Fig. 5. Each new fringe maximum corresponds to an additional $y$ displacement of $2 \mathrm{~mm}$. The $y$ displacement could be measured to within $0.1 \mathrm{~mm}$ by counting fractional fringes. The tension does not rise significantly until the string has deflected at least 3 or $4 \mathrm{~mm}$. The tension reaches its peak value a few $\mathrm{ms}$ before the string is displaced its maximum distance, because the rate at which the tension drops due to

Figure 5 Tension vs. time for a polyester string subject to the first of 10 impacts. Also shown is the corresponding laser signal used to measure the hammer speed and string displacement.

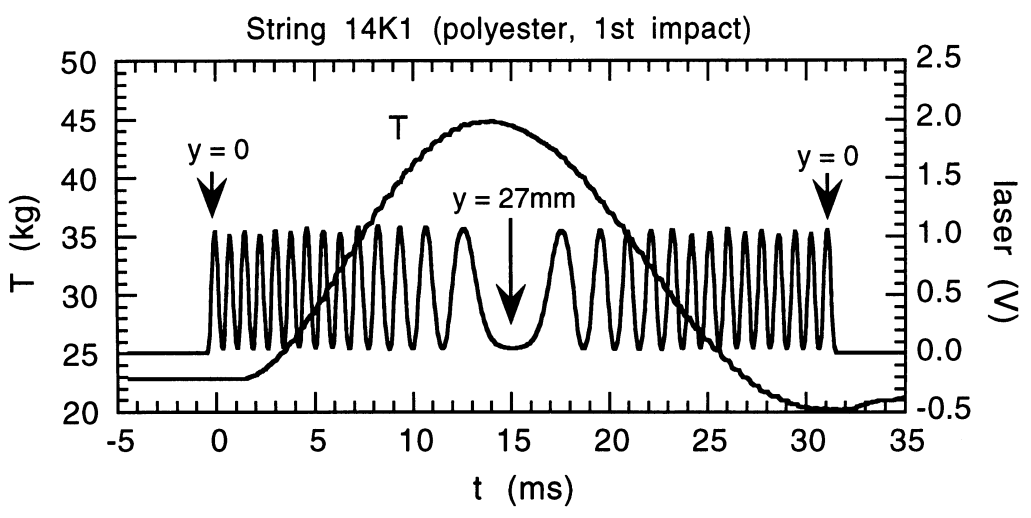


the breaking of molecular bonds is a significant fraction of the rate at which the tension increases due to the additional stretch. This is particularly noticeable during the first few impacts and with strings such as polyester where bonds are easily broken. The laser signal in Fig. 5 reveals that the rebound speed of the hammer is about $92 \%$ of the incident speed. This result was typical of the first few impacts for all strings. After the first few impacts, the rebound speed increased to between $95 \%$ and $98 \%$ of the incident speed for all strings, indicating that the energy loss in all strings is very small. One nylon string was tested with 200 impacts. Despite a significant loss in tension after 200 impacts, the energy loss in the string during each impact remained negligible, indicating no loss in resilience.

\section{Impact dynamics}

The deflection of a string at its midpoint is shown in Fig. 6. If the string has an initial length $L_{0}$ at tension $T_{0}$, and if the length increases to $L$ and the tension increases to $T$ when the string is displaced a distance $y$ at its midpoint, then

$$
L=\left(L_{0}^{2}+4 y^{2}\right)^{1 / 2}
$$

and

$$
T=T_{0}+k\left(L-L_{0}\right)
$$

where $k$ is the spring constant of the string. In general, $k$ is inversely proportional to $L_{0}$, it depends on the string type and the string diameter and it is also a function of both $T$ and $T_{0}$. The increase in the percentage elongation, $\Delta e$, above that required to stretch the string to a tension $T_{0}$, is given by

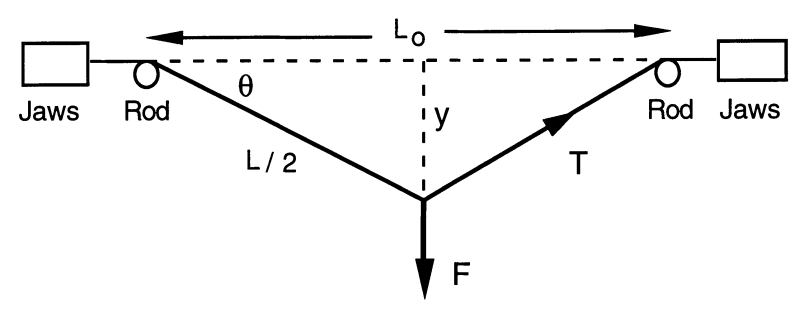

Figure 6 Geometry of a string deflected by a distance $y$ at the centre.

$$
\Delta e=100\left(L-L_{0}\right) / L_{0}
$$

The restoring force, $F$, acting at the midpoint is

$$
F=2 T \sin \theta=4 T y / L
$$

and the stiffness of the string in a direction perpendicular to the string is given by

$$
k_{\perp}=F / y=4 T / L
$$

In all cases of practical interest, $y$ is much smaller than $L_{0}$ in which case $L \approx L_{0}+2 y^{2} / L_{0}$, $T \approx T_{0}+2 k y^{2} / L_{0}$ and

$$
k_{\perp} \approx\left(4 / L_{0}\right)\left(T_{0}+2 k y^{2} / L_{0}\right)
$$

For small values of $y$, where $2 k y^{2} / L_{0} \ll T_{0}, k_{\perp}$ depends only on $T_{0}$ and $L_{0}$ and is independent of $k$. The perpendicular stiffness would then be same for all strings of the same length and initial tension, even if they were made from steel. Differences in $k_{\perp}$ only become apparent for relatively large values of $y$. Even then, the differences are not as large as one might expect. For example, if a mass impacts on a string with a large value of $k$, then $k_{\perp}$ will be relatively large, but the $y$ deflection will be relatively small. Inspection of Eq. (6) indicates that $k_{\perp}$ is not simply proportional to $k$ and that there is a possibility that $k_{\perp}$ could be almost independent of $k$ if $k y^{2}$ remains approximately constant. Similarly, $k_{\perp}$ could also be independent of $T_{0}$ if the increase in $y$ at low $T_{0}$ is large enough. The impact dynamics can be determined by solving the equation of motion for a mass $M$ impacting on the string in the $y$ direction at initial speed $v_{1}$. The equation of motion is then given by

$$
\mathrm{d}^{2} y / \mathrm{d} t^{2}=-F / M=-4 T y /(M L)
$$

assuming that there is no energy loss in the string. Numerical solutions of Eq. (7) are shown in Fig. 7 for a case where $M=0.45 \mathrm{~kg}, L_{0}=0.3 \mathrm{~m}$ and $v_{1}=2.63 \mathrm{~m} \mathrm{~s}^{-1}$, corresponding to the experimental conditions described above. Results are shown for three different values of $T_{0}$ and for a range of $k$-values from 10 to $150 \mathrm{kN} \mathrm{m}^{-1}$. Of all the strings tested, natural gut had the lowest $k$, about $20 \mathrm{kN} \mathrm{m}^{-1}$, and kevlar had the highest $k$, about $140 \mathrm{kN} \mathrm{m}^{-1}$. For these parameters, all strings 

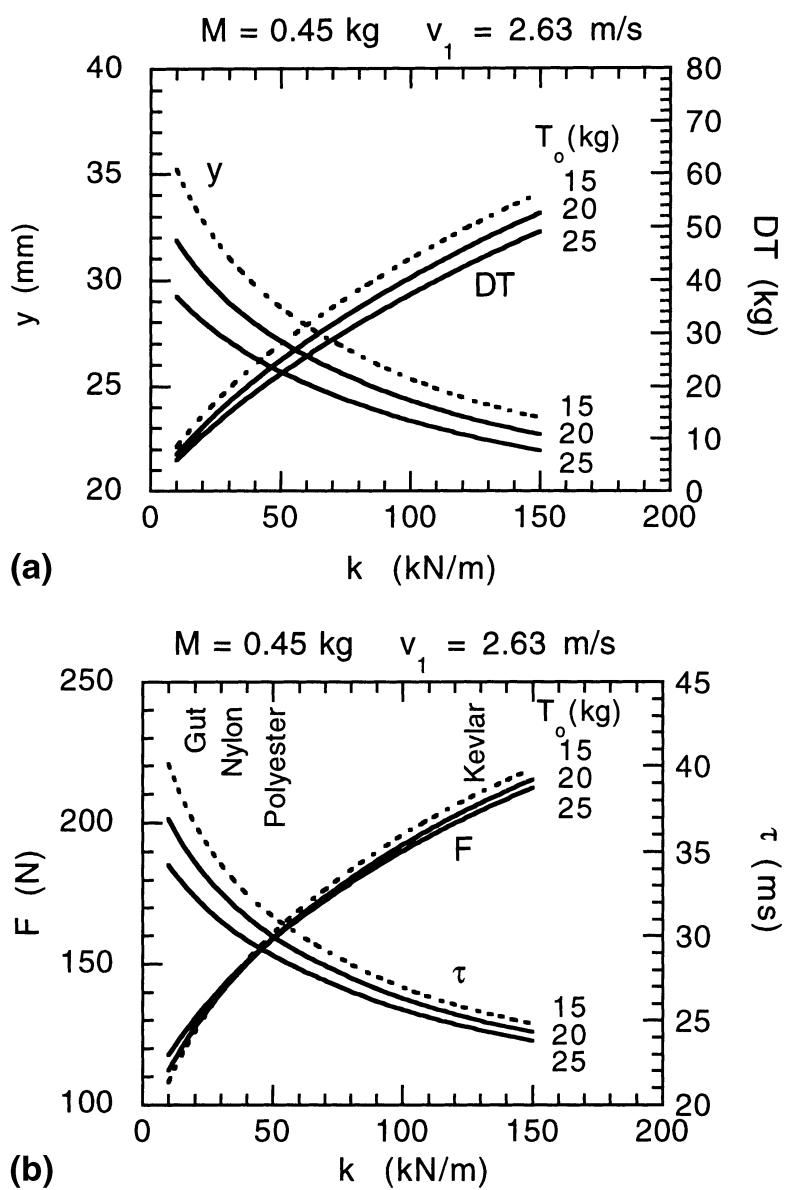

Figure 7 Numerical solutions of Eq. (7) for strings at an initial tension $T_{0}=15,20$ or $25 \mathrm{~kg}$, showing (a) the maximum string deflection $(y)$ and the increase in string tension (DT); (b) the peak force $(F)$ and the impact duration $(\tau)$.

deflect between 22 and $33 \mathrm{~mm}$ and the impact duration, $\tau$, varies from 24 to $36 \mathrm{~ms}$. The increase in tension, DT, is smallest for natural gut and largest for kevlar. As a result, the peak force on the string (or on the hammer) is smallest for gut and largest for kevlar. For any given $k$, the peak force is almost independent of the initial string tension. The total impulse on the hammer, $\int F \mathrm{~d} t$, is the same for all impacts because the rebound speed of the hammer is the same as the incident speed in this model. The force waveform is roughly a half sine wave at low $k$, but is more nonlinear at large $k$, having a narrower and more peaked or bell-shaped waveform.

\section{Analysis of selected impacts}

Analysis of the data in Fig. 5 reveals small departures from the behaviour of an ideal, lossless string. The example shown in Fig. 5 is somewhat atypical in that the tension loss during the impact is relatively large. As a result, there is a measurable loss of energy during the impact, which can be estimated from a plot of $F$ vs. $y$ as shown in Fig. 8. The $F$ waveform was extracted from the experimental $T$ and $y$ waveforms by means of Eq. (4). The integral $\int F \mathrm{~d} y$ from $y=0$ to the maximum value of $y$ represents the work done on the hammer in bringing it to rest, and is therefore equal to $M v_{1}^{2} / 2$. The value of this integral was $1.63 \pm 0.002 \mathrm{~J}$ for all impacts, and the incident hammer speed was $2.63 \mathrm{~m} \mathrm{~s}^{-1}$ for all impacts, indicating that the effective mass, $M$, of the hammer is $0.45 \mathrm{~kg}$. The integral $\int F \mathrm{~d} y$ during the rebound period represents the rebound energy
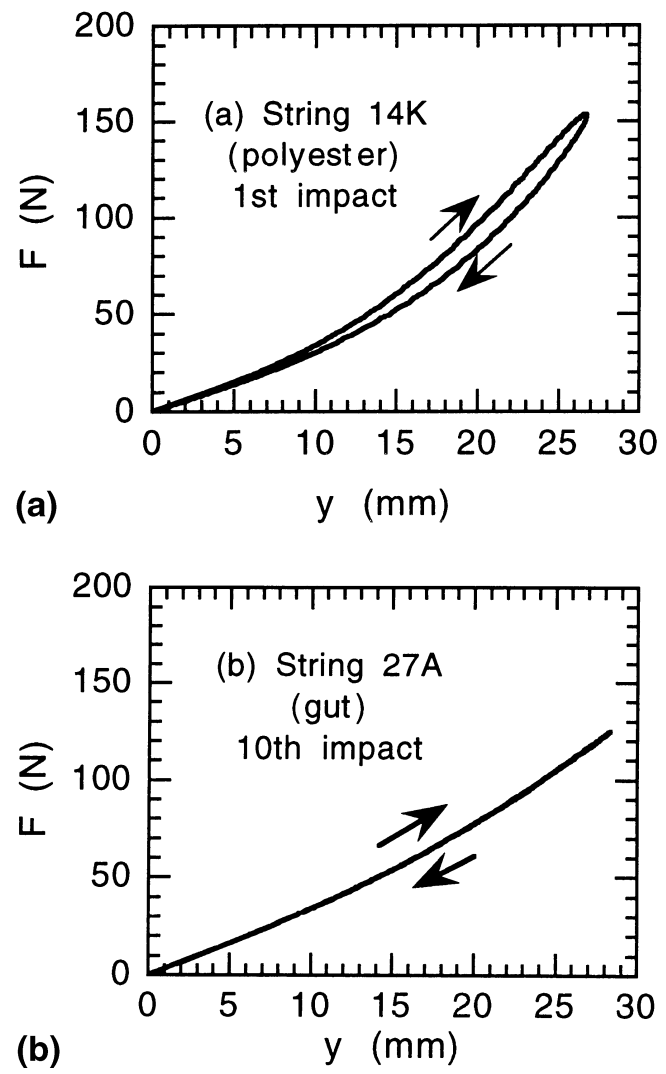

Figure $8 F$ vs. $y$ for (a) the impact shown in Fig. 5 and (b) a natural gut string. 
$M v_{2}^{2} / 2$. The integral over the entire impact period is represented by the area enclosed by the hysteresis loop and is equal to the energy loss in the string. In Fig. 8 , the area of the loop is $0.183 \mathrm{~J}$, and $v_{2} / v_{1}$ should equal 0.94 if there are no other energy losses. In fact, $v_{2} / v_{1}=0.92$ for this impact, indicating that there is an additional small loss in energy, presumably arising from vibrations in the hammer and its support structure. Vibrations in the support structure were minimised by bolting it to the wall. In cases where the tension loss during an impact was negligible, the observed ratio of $v_{2} / v_{1}$ was 0.98 . An example is shown in Fig. 8(b), corresponding to the 10th impact for natural gut.

In real strings, the percentage elongation also exhibits hysteresis, as shown in Fig. 9. Consequently, the spring constant, $k$, also exhibits hysteresis. This effect is significant only during the first few impacts when the tension loss is relatively large. For the remaining impacts, and even for the first few impacts, an average $k$ can be defined in terms of the peak tension and the peak elongation or the peak $y$ displacement, using Eq. (2). Alternatively, if one uses the approximations leading to Eq. (6), then $k=L_{0} \mathrm{DT} /\left(2 y_{0}^{2}\right)$, where $\mathrm{DT}$ is the maximum increase in tension during an impact and $y_{0}$ is the maximum $y$ displacement. The variation in $k$ from the first to the tenth impact was typically less than $3 \%$. Consequently, the dynamic $k$-values given below are quoted as the average over all 10 impacts. Similarly, the peak force, $F_{0}$, during each impact is given to a good approximation by $F_{0}=$ $4\left(T_{0}+\mathrm{DT}\right) y_{0} / L_{0}$. The peak force also varied by less than $3 \%$ from the first to the last impact, and is quoted below as the average over all 10 impacts.

\section{Summary of results}

The individual test results for all 90 strings were published in the USRSA Racquet Tech magazine, in June 2000. A summary of the results is given in Figs 10-12. Figures 10 and 11 can be compared directly with the theoretical calculations in Fig. 7. The scatter in the summarised data is due partly to the fact that the initial tension $T_{0}$ was not the same for every string or every impact. Additional scatter

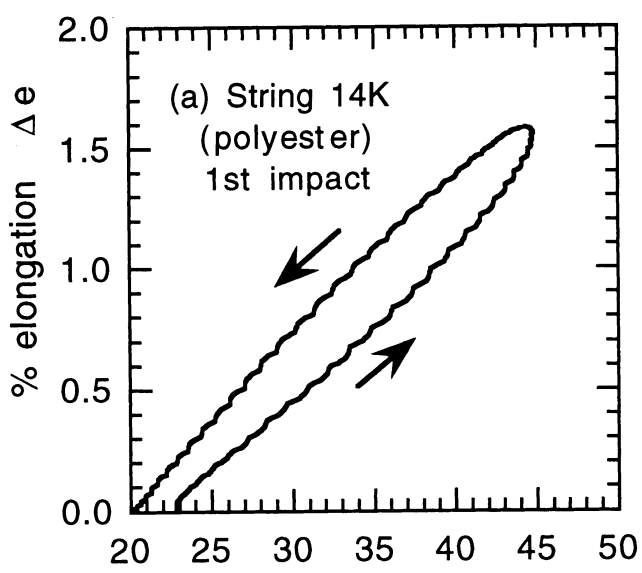

(a)

$\mathrm{T}(\mathrm{kg})$

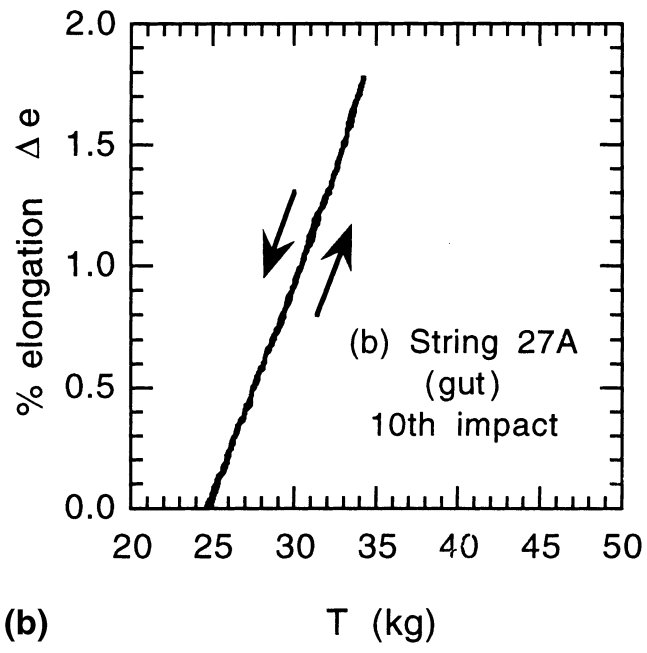

Figure 9 Increase in percentage elongation, $\Delta e$, vs. $T$ for the impact shown in Fig. 5.

results from the fact that the $k, y$ and DT measurements were averaged over 10 separate impacts. There is considerable overlap in the data points around $k=30-40 \mathrm{kN} \mathrm{m}^{-1}$ since the bulk of the strings tested were nylon strings having similar $k$-values. Only two natural gut strings were tested, both having $\mathrm{k} \sim 20 \mathrm{kN} \mathrm{m}^{-1}$. Most of the polyester strings had $k$-values between 40 and $60 \mathrm{kN} \mathrm{m}^{-1}$, while most of the kevlar strings had $k$-values from 90 to $140 \mathrm{kN} \mathrm{m}^{-1}$. Gut and nylon strings elongated by about $7-15 \%$ when tensioned to $28 \mathrm{~kg}$. Polyester strings elongated by about $4 \%$ and kevlar strings elongated by only 1 or $2 \%$. The elongation measurements are qualitatively consistent with the 


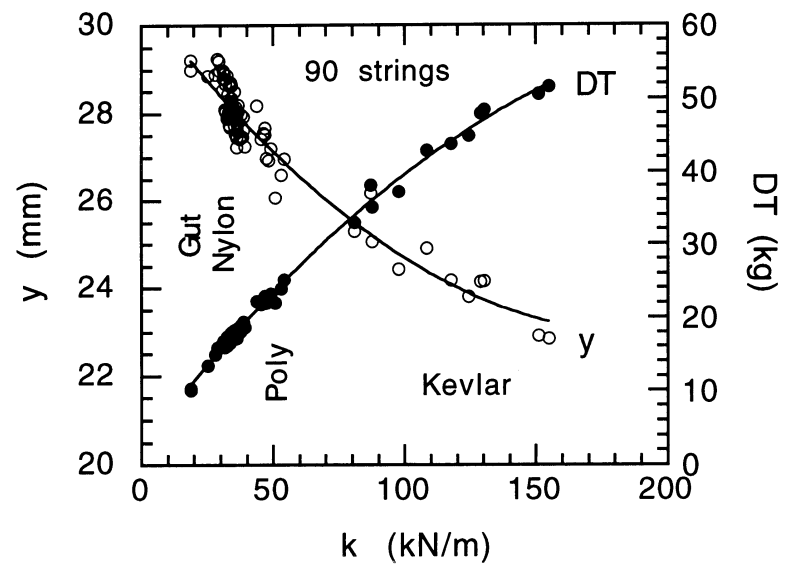

Figure 10 Results showing the maximum $y$ deflection and the increase in tension, DT, vs. dynamic stiffness, $k$, for 90 different strings subject to 10 impacts with a hammer. $y$, DT and $k$ are all averaged over the 10 impacts.

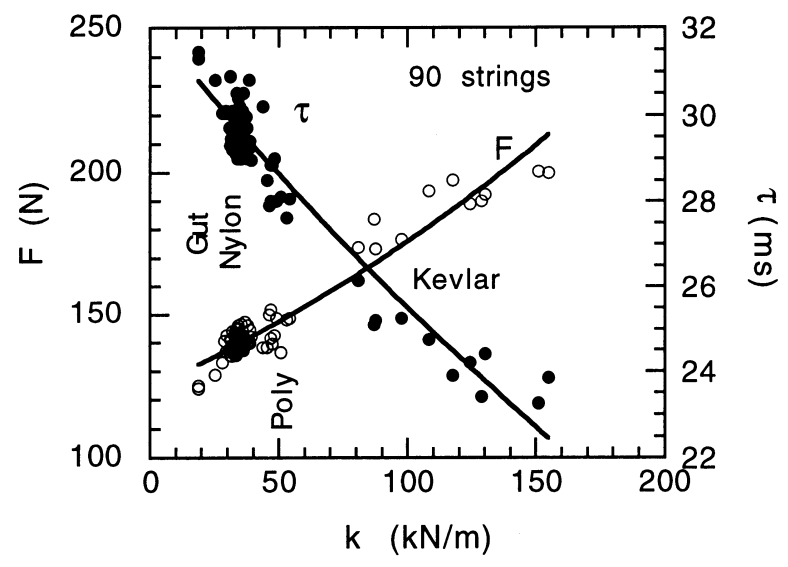

Figure 11 Results showing the measured impact duration, $\tau$ and the calculated peak force, $F$, for the same impacts as those in Fig. 10. $F$ and $\tau$ are averaged over the 10 impacts. $\tau$ was measured from the full width of $T$ vs. $t$ curves such as the one in Fig. 5. The full width of the $y$ vs. $t$ curves is typically $2 \mathrm{~ms}$ longer.

dynamic $k$ results, but a direct comparison is not meaningful since elongation curves are usually nonlinear functions of both time (due to creep) and string tension. As a result of creep, quasi-static estimates of $k$ at any given tension are typically a factor of between 1.5 and 2 times lower than dynamic measurements of $k$, at least on a fresh, previously unstretched string. Elongation measurements undertaken immediately after each string

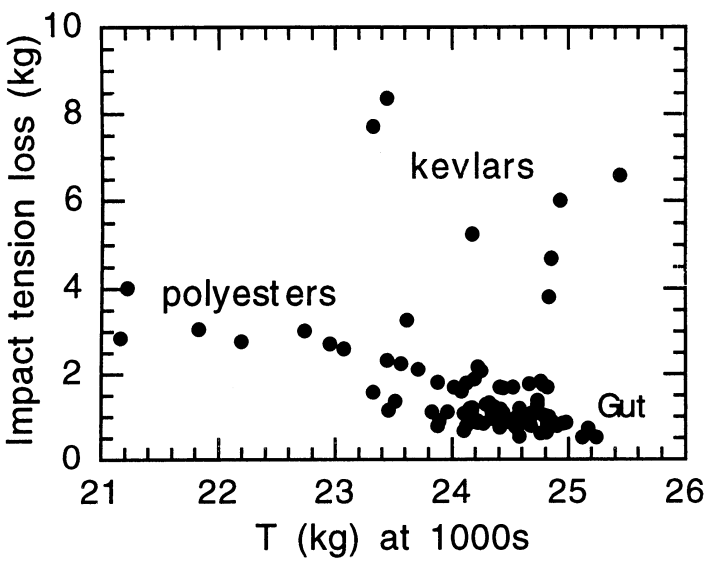

Figure 12 The total decrease in tension resulting from 10 impacts, as a function of the tension recorded $1000 \mathrm{~s}$ after clamping, for the same impacts as those in Fig. 10. All strings were tensioned to $28 \mathrm{~kg}$ for $10 \mathrm{~s}$ prior to clamping.

had been subjected to 10 impacts gave much closer agreement between static and dynamic measurements of $k$, typically within $10-20 \%$.

Figure 12 shows the net loss in tension resulting from the 10 impacts as a function of the tension $1000 \mathrm{~s}$ after clamping the string. It is reasonable to assume that a string with strong molecular bonds will lose tension slowly with both time and repeated impacts. The majority of strings fit this description, but kevlar strings are exceptional. Most of the kevlar strings lost tension slowly with time but the tension loss during each impact was larger than that for other strings. The low loss in tension with time is observed at tensions up to $28 \mathrm{~kg}$. The high loss in tension due to each impact is related to the fact that the increase in tension, DT, during each impact is much larger than the rise in other strings. Tests at lower hammer speeds indicated that for any given hammer speed, the impact tension loss in a kevlar string is always larger than the loss in other strings and that the tension loss is roughly proportional to the hammer speed.

\section{Coefficient of friction}

When a ball impacts obliquely on the strings of a racket, the rebound angle depends on the coefficient of friction between the ball and the strings, 
for the same reason that the rebound angle off the court surface depends on whether the court is fast or slow (Cross, 2000b). The coefficient of friction between the ball and the strings has not yet been measured very precisely, but it is likely to depend on a number of factors such as the number and diameter of strings, as well as the surface roughness and composition of individual strings. With topspin or backspin, the ball generally slides along the cross strings and across the main strings. The coefficient of friction for a ball that is dragged along a set of parallel strings glued to a flat surface was measured and found to be significantly lower than the coefficient of friction when the ball is dragged across the same set of strings. This experiment is still in progress, but it is clear that both coefficients increase as the surface roughness of the strings increase, and both contribute to the total friction force on the ball. Consequently, it was decided to include some simple measurements of string friction when comparing the 90 different strings. The procedure, as described below, measures the coefficient of friction along rather than across the strings.

Measurements of the coefficient of sliding friction between a string and the cloth material of a tennis ball were made by gluing tennis ball cloth to a 60-mm diameter, $150-\mathrm{mm}$ long PVC pipe so that the cloth completely covered the exterior surface of the pipe. The pipe was mounted horizontally so that it could not move or rotate. Two turns of a one metre length of string were wrapped around the cloth, a mass $M=0.15 \mathrm{~kg}$ was clamped onto the lower end of the string, and the upper end was attached to a spring balance. The force required to lift the mass vertically at a constant low speed was measured by the spring balance. The required force varied from $0.6 \mathrm{~kg}$ to about $10 \mathrm{~kg}(98 \mathrm{~N})$, depending on the coefficient of friction between the string and the cloth. The force, $F$, required to lift the mass is given by

$$
F=(M g) e^{2 \pi \mu N}
$$

where $N$ is the number of turns and $\mu$ is the coefficient of sliding friction. Most strings had values of $\mu$ in the range $0.15-0.18$. Some polyester strings had $\mu$ values as low as 0.11 . A small number of strings had a larger surface roughness or a different texture, with $\mu$ values in the range $0.2-0.36$.

String friction affects not only the rebound angle but it is also likely to affect string tension. When a stringer strings a racket, one part of the string is held in a clamp and the free end is pulled through grommet holes. Friction between the string and the grommet holes will result in different tensions between the clamped end and the pulled end, as described by Eq. (8) with $N=0.5$. From this point of view, strings with low $\mu$ are likely to be at a more even and higher overall tension while strings with large $\mu$ will provide a better grip on the ball.

\section{Discussion}

The best string to use in a tennis racket is the one that the player likes best, but many of the top professional players prefer natural gut. Gut is a highly elastic string and it maintains tension better than most other strings. A significant number of top players actually prefer strings such as polyester which have the opposite properties. Polyester is a relatively stiff string, and it loses tension relatively quickly. It appears that the two undesirable properties of polyester might tend to combine to produce a good string. The tension drops rapidly, immediately after a racket is strung with a polyester string and it drops significantly with repeated impacts. The tension increases substantially during each impact with a ball, due to the high stiffness of polyester. The average tension during an impact with a polyester string may then be similar to that with a gut string. However, even if the average tension is the same, the force and the impact duration are not necessarily the same. As shown in Fig. 7(b), the force on a gut string will always be smaller than the force on a polyester string (for the same racket, the same number and gauge of strings and the same impact speed) regardless of the initial tension. Similarly, the impact duration is usually longer on gut than polyester, although the duration may be almost the same if gut is at quite high initial 
tension and polyester is at quite a low initial tension.

The magnitude, as well as the duration of the force acting on the strings of a racket will affect the feel of the strings, and it will also affect ball control. The 'feel' is not a quantity that is measurable with instruments available to scientists, but the physical effects of the force waveform are clear. For example, a large force on the strings will generate a large force acting on the hand, at least for impact points well removed from the sweet spot area of the strings. For an impact at the sweet spot, the force on the hands and the arm is much reduced (Cross 1999), in which case all strings are likely to feel much the same. If the total impulse (i.e. the time integral of the force) is held constant, then the effect of a large force acting for a short time might be expected to have the same effect as a small force acting for a longer time. However, there are two significant differences. First, a short duration impact will excite high frequency vibrations of the racket frame more efficiently than a long duration impact (Cross 2000c). The second difference is that, for a long duration impact, the racket will rotate through a larger angle while the ball is in contact with the strings. For example, if the average angular velocity of the racket during the impact is say $20 \mathrm{rad} \mathrm{s}^{-1}$, then the racket will rotate through an angle of $5.7^{\circ}$ during a 5 -ms impact, or by $6.9^{\circ}$ for a 6-ms impact. A change in impact duration will therefore result in a different rebound angle of the ball. Similarly, if the ball impacts the strings at a point towards the edge of the racket frame, the racket will rotate about the long axis through the handle, and the ball will be deflected away from the desired trajectory. For a given impulse, the rotation angle is proportional to the impact duration (Cross 2000c). Consequently, some players may prefer polyester strings since the impact duration is shorter and hence ball control is improved, while others may prefer gut since it has a softer feel. In either case, the energy loss in the string is usually negligible, and there should be almost no difference in the speed of a ball rebounding off gut or polyester strings, despite the slightly enhanced losses in the ball and the slightly enhanced frame vibration losses when a ball impacts on stiff strings (Cross 2000c). For many players, nylon strings seem to offer the best compromise in terms of feel, control, price and durability. Gut and polyester strings are not noted for their durability.

\section{Conclusions}

The test methods described in this paper show that tennis strings can be categorised by dynamic stiffness, by tension loss with time and with repeated impacts and by the coefficient of friction between the string and the ball. The energy loss in a string is too small to make any significant difference in resilience between different strings, even after many impacts. There are four broad types of string making up the bulk of strings available on the market. In order of increasing dynamic stiffness, these types are natural gut, nylon, polyester and kevlar. Only one zyex string was available for testing. It's dynamic stiffness was between that of natural gut and nylon. The most common type of string on the market is nylon, which is available in many forms depending on whether it is constructed from a solid core, whether it contains many small diameter filaments and whether it contains other composite materials or resins or coatings. The different construction methods for nylon account for a small spread in properties, but there is no significant overlap with either natural gut or polyester which are distinctly different. Kevlar strings are also very distinctive in that they are very stiff and they suffer a larger impact tension loss than other strings. From this point of view, it is difficult to compare one nylon string with another. All nylon strings have similar physical properties. The differences between any two nylon strings are relatively small compared with the relatively large differences between nylon and other types of string.

The results presented in this paper are the first to categorise a significant sample of tennis strings using measurements of physical properties. Further work is needed to relate these properties to players' perceptions of the strings, to compare the 
properties of a single string to the behaviour in a fully strung racket, and to extend the measurements to include other strings, other string diameters and other impact conditions.

\section{Acknowledgements}

This work was funded partly by the USRA (United States Racquet Stringers Association) and was made possible by the cooperation of the companies who provided the strings, namely Alpha, Ashaway, Babolat, Bow Brand, Forten, Gamma, Gosen, Head, Klip, Prince, Tecnifibre, Velociti, Wilson and Yonex.

\section{References}

Brody, H. (1987). Tennis Science for Tennis Players. University of Pennsylvania Press, Philadelphia, PA, USA.
Calder, C.A., Holmes, J.G. \& Mastry, L.L. (1987) Static and dynamic characteristics of tennis string performance, ITF technical report ITF-L-015. International Tennis Federation, London, UK.

Casolo, F. \& Lorenzi, V. (2000) On tennis equipment, impact simulation and stroke precision. Tennis Science and Technology (eds Haake, S.J. \& Coe, A.O.). Blackwell Science, Oxford, UK, pp. 83-90.

Cross, R.C. (1999) The sweet spots of a tennis racket. Sports Engineering, 1, 63-78.

Cross, R.C. (2000a) Physical Properties of Tennis Strings, 3rd ISEA International Conference on the Engineering of Sport, Sydney (eds Subic, A.J. \& Haake, S.J.). Blackwell Science, Oxford, UK, pp. 213-220.

Cross, R.C. (2000b) Effects of friction between the ball and strings in tennis. Sports Engineering, 3, 85-97.

Cross, R.C. (2000c) Flexible beam analysis of the effects of string tension and frame stiffness on racket performance. Sports Engineering, 3, 111-122. 